\title{
Impact of the STAT1 N-terminal domain for fibrosarcoma cell responses to $\gamma$-irradiation
}

\author{
Anja Göder ${ }^{1} \uparrow$, Torsten Ginter ${ }^{2}$, Ulrike Kröhnert ${ }^{2}$, Christian Kosan $^{2}$ and Oliver H. Krämer ${ }^{1, *}$ (D) \\ ${ }^{1}$ Department of Toxicology, University Medical Center, Obere Zahlbacher Str. 67, 55131 Mainz, Germany, and ${ }^{2}$ Center for \\ Molecular Biomedicine (CMB), Institute for Biochemistry, Friedrich-Schiller University Jena, Hans-Knöll Str. 2, 07745 Jena, \\ Germany \\ ${ }^{\star}$ Corresponding author: Email: okraemer@uni-mainz.de \\ †present address: Centre for Chromosome Biology, School of Natural Sciences, National University of Ireland Galway, Ireland
}

(Received 25 January 2020; Revised 01 June 2020; Accepted 03 June 2020)

\begin{abstract}
Type I/II interferons (IFN $\alpha, \beta / \mathrm{IFNy}$ ) are cytokines that activate signal-transducer-and-activator-oftranscription-1 (STAT1). The STAT1 N-terminal domain (NTD) mediates dimerization and cooperative DNA-binding. The STAT1 DNA-binding domain (DBD) confers sequence-specific DNA-recognition. STAT1 has been connected to growth inhibition, replication stress and DNA-damage. We investigated how STAT1 and NTD/DBD mutants thereof affect fibrosarcoma cells. STAT1 and indicated mutants do not affect proliferation of resting and IFN $\alpha$-treated cells as well as checkpoint kinase signaling, and phosphorylation of the tumor-suppressive transcription factor p53 ensuing $\mathrm{\gamma}$-irradiation. Of the STAT1 reconstituted U3A cells those with STAT1 NTD mutants accumulate the highest levels of the replication stress/DNAdamage marker S139-phosphorylated histone H2AX ( $\mathrm{\gamma H} 2 \mathrm{AX}$ ). This is similarly seen with a STAT1 NTD/DBD double mutant, indicating transcription-independent effects. Furthermore, U3A cells with STAT1 NTD mutants are most susceptible to apoptotic DNA fragmentation and cleavage of the DNA repair protein PARP1. These data provide novel insights into the relevance of the STAT1 NTD.
\end{abstract}

Keywords: STAT1; dimer; DNA-damage; checkpoint kinase signaling

\section{Highlights:}

- STAT1 and NTD mutants can be stably expressed in U3A cells

- Similar effects of wild-type and mutant STAT1 on proliferation \pm IFNa

- $\mathrm{STAT}^{\mathrm{FA}}$ accentuates $\mathrm{\gamma H} 2 \mathrm{AX}$ accumulation and apoptosis upon $\gamma$-irradiation

\section{Introduction}

Binding of cytokines and growth hormones to cognate receptors activates the inducible transcription factors STAT1,-2,-3,-4,-5A,-5B,-6. STATs have a modular structure (NTD, coiled-coil, DBD, linker, SH2-domain, p-tyrosine site, C-terminal activation domain) (Khodarev et al., 2012; Wieczorek et al., 2012). STAT1 phosphorylation at Y701 leads to high affinity interactions with other STATs. The NTD allows cooperative STAT1-STAT1/STAT1-STAT2 DNA-binding of pre-formed dimers and enables STAT1 dephosphorylation (Mertens et al., 2006; Wieczorek et al., 2012).

\footnotetext{
() The Author(s), 2020. Published by Cambridge University Press. This is an Open Access article, distributed under the terms of the Creative Commons Attribution licence (http://creativecommons.org/licenses/by/4.0/), which permits unrestricted re-use, distribution, and reproduction in any medium, provided the original work is properly cited.
} 
IFN-/STAT1-related signatures promote resistance to chemotherapy and $\gamma$-irradiation in cancer cells (Ah-Koon et al., 2016; Kaowinn et al., 2017; Khodarev et al., 2012; Malilas et al., 2013). Intriguingly, STAT1 can though promote sensitivity to DNA crosslinking agents (Prieto-Remon et al., 2013) and proapoptotic gene expression (Khodarev et al., 2012; Wieczorek et al., 2012). Hence, modulators of STAT1 may be novel and innovative context-dependent anti-cancer drugs (Khodarev et al., 2012).

\section{Objective}

It is unclear whether the STAT1 NTD and DBD modulate cellular responses to fibrosarcoma cell growth and their responses to IFN $\alpha$ and $\gamma$-irradiation. We set out to answer these questions. We reconstituted STAT1 negative U3A fibrosarcoma cells with wild-type STAT1 and NTD/DBD mutants thereof. We investigated cellular responses and molecular parameters.

\section{Material and Methods}

Cell lines

Cells were cultured as described (Ginter et al., 2012) and table 1.. See figure legends for $\gamma$-irradiation.

\section{Plasmids}

We refer to HA-tagged STAT1 $\alpha$ as STAT1. STAT1AA has moieties phenylalanine-77 and leucine-78 replaced by alanine moieties (mutagenic primers 5-GCTTTTCTTTGGAGAATAACGCCGCGC TACAGCATAACATAAGG-3/5-CCTTATGTTATGCTG TAGCGCGGCGTTATTCTCCAAAGAAAA GCG-3). STAT1AAQQ carries these mutations and additional lysine- 410 and lysine- 413 exchanges to glutamine residues (Ginter et al., 2012).

\section{Western blot, immunoprecipitation, antibodies, drugs, chemicals, cytokines}

For Western blot technique see (Beyer et al., 2017). Antibodies: Santa-Cruz-Biotechnology ( $\beta$-actin/sc47,778;p53/sc-81,168;IFNAR/sc-845; STAT1/sc-346/sc-417; p-STAT1/sc-7,988-R); Sigma (tubulin- $\alpha /$ T5168); Abgent (UBCH8/AP2118b); BD-Pharmingen (cleaved PARP1/552596); Abcam (ATM/ab32420;p-S1981-ATM/ab81292); Cell-Signalling (ATR/2790;CHK1/2360;p-S317-CHK1/2344;

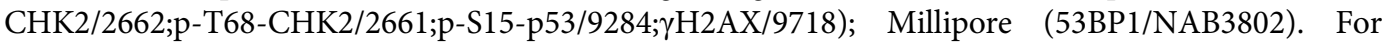
immunoprecipitation, drugs, chemicals, and cytokines see (Ginter et al., 2012). Immunoblot data show results from at least two independent experiments.

\section{Cell cycle and apoptotic DNA fragmentation analyses}

Cells were incubated with $10^{3} \mathrm{U} / \mathrm{ml}$ IFN $\alpha$ or $10 \mathrm{~Gy}$ using a Gammacell 2000 irradiator. Adherent and floating cells were collected. Single-cell suspensions in PBS were centrifuged ( $5 \mathrm{~min}, 700 \mathrm{xg}$, RT). Pellets

Table 1.

\begin{tabular}{lll}
\hline U3A reconstituted with & Resistance & STAT1 \\
\hline GFP & Neomycin (G418) & Null \\
\hline STAT1 & Neomycin (G418) & Wild-type \\
\hline STAT1AA & Neomycin (G418) & NTD mutant \\
\hline STAT1QQ & Neomycin (G418) & DBD mutant \\
\hline STAT1AAQQ & Neomycin (G418) & NTD/DBD mutant \\
\hline
\end{tabular}


A

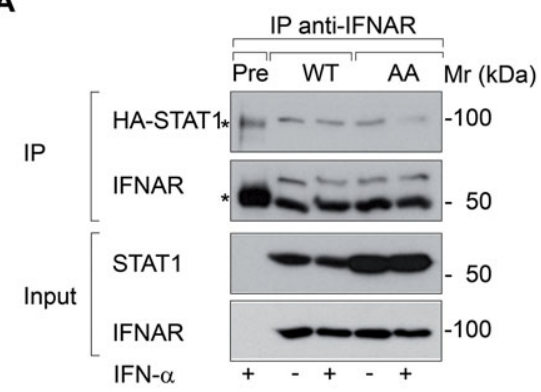

C
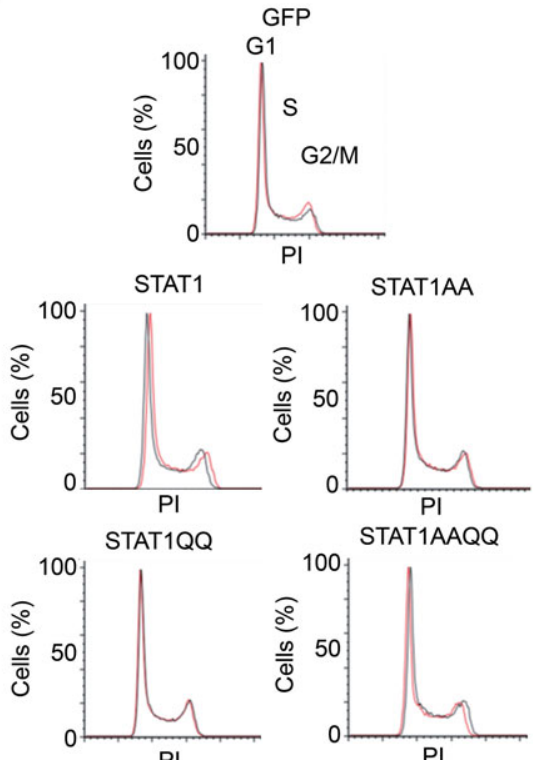

B

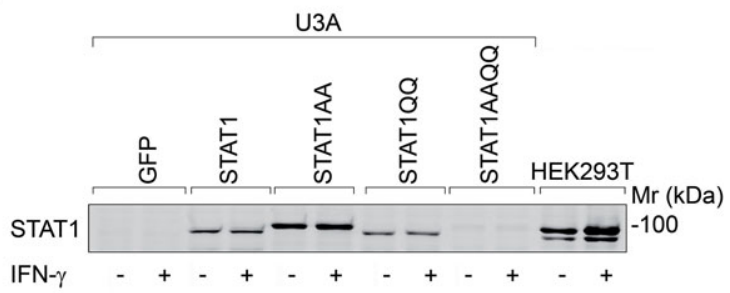

D

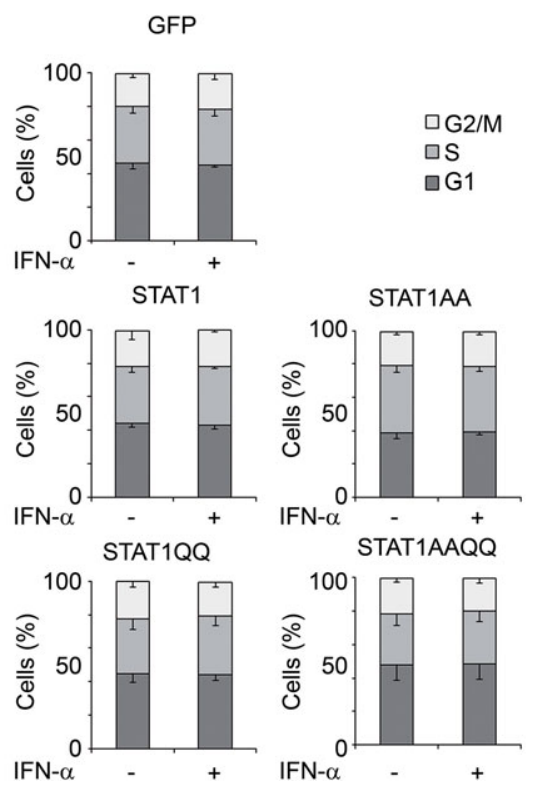

Figure 1. IFNs do not restrict the growth of fibrosarcoma cells \pm STAT1. (A) HA-tagged STAT1 or STAT1AA were transfected into HEK $293 \mathrm{~T}$ cells. $24 \mathrm{~h}$ later, the cells were stimulated with $1,000 \mathrm{U}$ IFN $\alpha$ for $1 \mathrm{~h}$. Lysates were collected and subjected to immunoprecipitation against IFNAR. Western blots were probed for immunoprecipitated IFNAR and bound HA-tagged STAT1 variants; * non-specific band resulting from IgG. (B) Detection of wild-type STAT1 and mutants thereof in reconstituted U3A fibrosarcoma cells. Equal amounts of lysates from untreated and IFNy-treated $(10 \mathrm{ng} / \mathrm{ml}, 24 \mathrm{~h})$ U3A cells were probed for STAT1 by Western blot. HEK 293 T cells served as controls expressing the endogenous STAT1 gene (encoding STAT1 $\alpha$ and STAT1 $\beta$ ) which is induced upon IFN treatment. (C) U3A cells stably expressing STAT1 (WT, wild-type), STAT1 GFP, STAT1K410/413Q (QQ) and/or STAT1F77A/L78A (AA) were treated with 1,000 U IFN $\alpha$ (+, red lines) or were kept untreated (-, black lines) for $96 \mathrm{~h}$. Cell cycle analysis was performed using propidium iodide (PI) staining on fixed cells. The cell cycle phases are marked for GFP-transfected cells as an example (G1, G1 phase; S, S phase; G2, G2/M phase). Graphs are representative for $n=3$.(D) Same as in (C), bar graphs show the average percentages of cells in $G 1, S$ and $G 2 / M$ cell cycle phase. Data represent three independent experiments, of which one is shown as (C). Error bars indicate standard deviations. No statistically significant differences were seen (t-test).

were fixed ( $1 \mathrm{ml}$ ice cold $70 \%$ ethanol), stored at $-20^{\circ} \mathrm{C}$ overnight, and washed in $1 \mathrm{ml}$ PBS (5 min at 700xg, RT). Per sample, $390 \mu \mathrm{l} \mathrm{PBS,} 5 \mu \mathrm{l}$ Rase A $(10 \mathrm{mg} / \mathrm{ml})$, and $5 \mu \mathrm{l}$ PI $(2.5 \mathrm{mg} / \mathrm{ml})$ were added $(20$ min, $\left.37^{\circ} \mathrm{C}\right)$. Samples were analyzed by flow cytometry.

\section{Results}

\section{STAT1 and NTD/DBD mutants do not impair growth}

We investigated if IFN $\alpha$-activated STAT1 has anti-proliferative effects in U3A cells. STAT1 (WT) and STAT1AA (lacks NTD for dimerization and cooperative DNA binding (Mertens et al., 2006)) bound to 
A

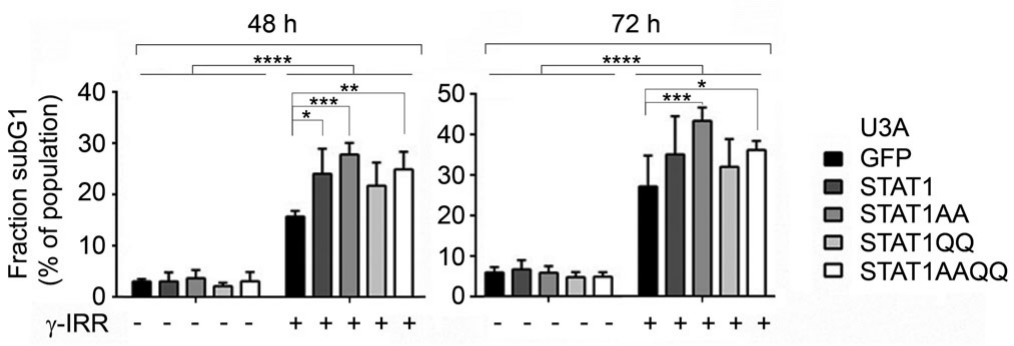

B

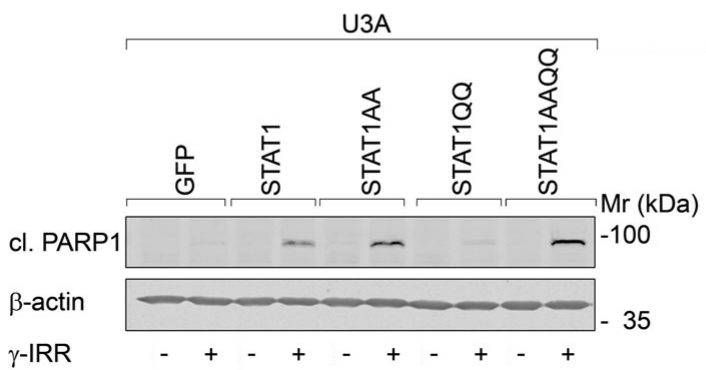

Figure 2. STAT1 sensitizes fibrosarcoma cells to cytotoxic effects of $\gamma$-irradiation. (A) U3A cells stably expressing STAT1, GFP, STAT1K410/413Q (QQ) and/or STAT1F77A/L78A (AA) were irradiated with $10 \mathrm{~Gy}$ ionizing radiation (+) or kept untreated (-). This was followed by an incubation at $37^{\circ} \mathrm{C}$ for $48 \mathrm{~h}$ or $72 \mathrm{~h}$. SubG1 fractions were assessed via flow cytometry and PI staining. Data represent three $(48 \mathrm{~h})$ or four $(72 \mathrm{~h})$ independent experiments. Two-way ANOVA, Šidák multiple comparisons test, error bars indicate standard deviations; ${ }^{\star} p<0.05$, ${ }^{\star \star} p<0.01$, ${ }^{\star \star \star} p<0.001$, ${ }^{\star \star \star \star} p<0.0001$. (B) U3A cells were treated as described in (A) for $72 \mathrm{~h}$. Whole cell extracts were prepared and indicated proteins were detected by SDS-PAGE and Western blot analysis. Both adherent and floating cells were harvested for immunoblot and flow cytometry $(n=2)$.

the IFN $\alpha$-receptor (IFNAR) (Fig. 1A). However, IFN $\alpha$ did not affect the cell cycle of U3A cells reconstituted with STAT1 or NTD (AA)/DBD (QQ) mutants (Table 1: and Fig. 1B-D).

\section{$\gamma$-irradiation differentially affects U3A cells with STAT1 and its mutants}

$\gamma$-irradiation evoked the accumulation of a subG1 fraction (Fig. 2A) and of cleaved PARP1 more pronouncedly in cells with STAT1AA and STAT1AAQQ (Fig. 2B). Apparently, y-irradiation causes apoptosis and STAT1 accentuates this.

\section{STAT1 NTD affects $y$ H2AX accumulation upon $\gamma^{\text {-irradiation }}$}

Next, we analyzed DNA damage sensors. 53BP1 levels were not correlated with STAT1 per se (Fig. 3A). Apical (ATM/ATR) and downstream checkpoint kinases (CHK1/CHK2) and their target p53 were phosphorylated after $\gamma$-irradiation irrespective of STAT1 (Fig. 3B, C). In contrast, the checkpoint kinase target $\mathrm{\gamma H}_{2} \mathrm{AX}$ accumulated most pronouncedly in U3A cells with STAT1AA/STAT1AAQQ (Fig. 3C). Hence, NTD mutant STAT1 expression correlates with irradiation-induced $\gamma \mathrm{H} 2 \mathrm{AX}$.

\section{Discussion}

STAT1 sensitizes fibrosarcoma cells to DNA damage and apoptosis upon $\gamma$-irradiation. Cooperativity through the STAT1 NTD seems to attenuate this. Thus, STAT1 signaling could be analyzed further as a target in fibrosarcoma and potentially other cancers. Fibrosarcoma is a clinically unmet problem with poor survival due to resistance to chemotherapy and irradiation as well as tumor recurrence after surgery. Novel treatments and the identification of mechanisms that regulate the therapeutic sensitivity of 
A

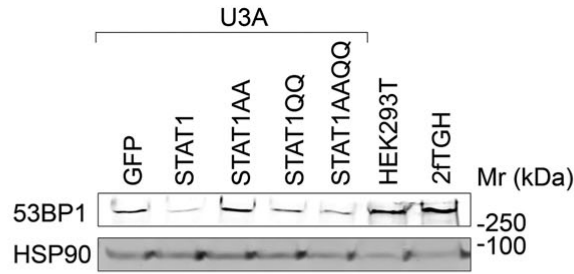

B

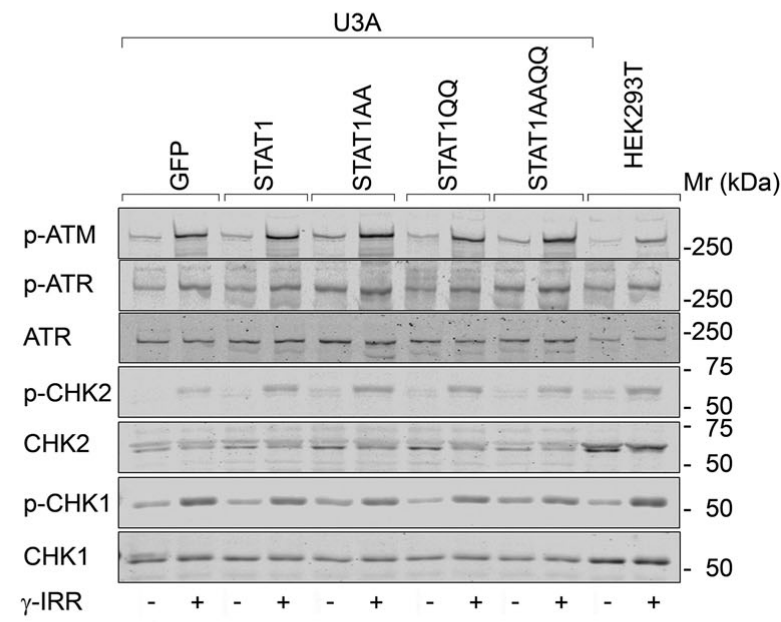

C

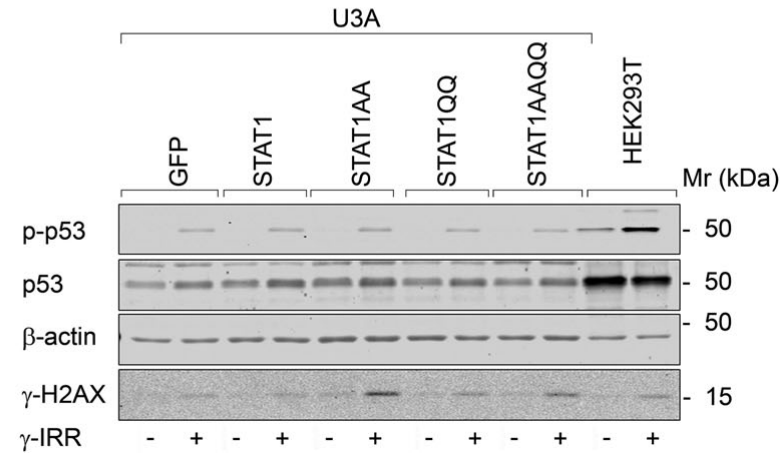

Figure 3. STAT1 does not alter checkpoint kinase signaling after y-irradiation. (A) 53BP1 is a key regulator of DNA doublestrand breaks processing and their repair by the non-homologous end-joining DNA repair pathway. U3A cells stably expressing STAT1, GFP, STAT1K410/413Q (QQ) and/or STAT1F77A/L78A (AA) were analyzed for 53BP1; HSP90 as loading control. 2fTGH cells (STAT1 positive parental cells for U3A cells) and HEK 293 T cells served as controls with endogenous STAT1. (B) U3A cells stably expressing STAT1, GFP, STAT1K410,413Q (QQ) and/or STAT1F77A, L78A (AA) and HEK293T cells were irradiated with $10 \mathrm{~Gy}$ ionizing radiation $(+)$ or kept untreated $(-)$. After $2 \mathrm{~h}$, cells were harvested, lysed and samples were analyzed via Western blot for checkpoint kinase phosphorylation (pS1981-ATM, pT68-CHK2, pS317-CHK1) and total levels $(n=4)$. (C) Same as in (B), but analysis of S15-phosphorylated and total p53 and $\gamma_{\mathrm{H}} 2 \mathrm{AX} ; \beta$-actin as loading control. Both adherent and floating cells were harvested for immunoblot $(n=3)$.

fibrosarcoma could solve the clinical problem (Augsburger et al., 2017). Perhaps, STAT1 is both a clinically applicable marker and novel agents targeting the STAT1 NTD might give therapeutic benefits.

\section{Conclusion}

Our work provides fresh information on the relevance of STAT1 and a limited number of NTD/DBD mutants thereof for cellular responses to IFN $\alpha$ and $\gamma$-irradiation. We speculate that the higher levels of 
$\mathrm{\gamma H} 2 \mathrm{AX}$ in U3A cells with STAT1AA/AAQQ result from attenuated DNA repair and/or apoptosis. It is plausible that the non-cooperative STAT1 NTD mutants have binding partners that are not engaged by the dimerization-competent wild-type STAT1. Future studies are needed to reveal if such proteins might be DNA repair proteins and/or transcription factors.

Acknowledgement. We thank Sigrid Reichardt for technical assistance and Prof. Dr. T. Heinzel for helpful discussions. Moreover, we are indebted to Prof. Dr. Darnell for the HA-STAT1 plasmid.

Funding Information. Funding was from intramural funds (University Medical Center Mainz and CMB Jena).

Conflicts of interest. All authors (A.G., T.G., U.K., C.K., OH.K.) declare no conflicts of interest.

Authors contributions. A.G., T.G., U.K. conducted data gathering.

A.G., C.K. performed statistical analyses.

A.G., O.H.K. conceived and designed the study.

OH.K. wrote the article.

Data availability statement. The data that support the findings of this study are available from the corresponding author, O.H.K., upon reasonable request.

\section{References}

Ah-Koon, L., Lesage, D., Lemadre, E., Souissi, I., Fagard, R., Varin-Blank, N., Fabre, E. E., \& Schischmanoff, O. (2016). Cellular response to alkylating agent MNNG is impaired in STAT1-deficients cells. Journal of Cell and Molecular Medicine, 20, 1956-1965.

Augsburger, D., Nelson, P. J., Kalinski, T., Udelnow, A., Knösel, T., Hofstetter, M., Qin, J. W., Wang, Y., Gupta, A. S., Bonifatius, S., Li, M., Bruns, C. J., \& Zhao, Y. (2017). Current diagnostics and treatment of fibrosarcoma-Perspectives for future therapeutic targets and strategies. Oncotarget, 8, 104638-104653.

Beyer, M., Kiweler, N., Mahboobi, S., \& Krämer, O. H. (2017). How to Distinguish Between the Activity of HDAC1-3 and HDAC6 with Western Blot. Methods in Molecular Biology, 1510, 355-364.

Ginter, T., Bier, C., Knauer, S. K., Sughra, K., Hildebrand, D., Münz, T., Liebe, T., Heller, R., Henke, A., Stauber, R. H., Reichardt, W., Schmid, J. A., Kubatzky, K. F., Heinzel, T., \& Krämer, O. H. (2012). Histone deacetylase inhibitors block IFNgamma-induced STAT1 phosphorylation. Cell Signalling, 24, 1453-1460.

Kaowinn, S., Jun, S. W., Kim, C. S., Shin, D.-M., Hwang, Y.-H., Kim, K., Shin, B., Kaewpiboon, C., Jeong, H. H., Koh, S. S., Krämer, O. H., Johnston, R. N., \& Chung, Y.-H. (2017). Increased EGFR expression induced by a novel oncogene, CUG2, confers resistance to doxorubicin through Stat1-HDAC4 signaling. Cellular Oncology, 40, 549-561.

Khodarev, N. N., Roizman, B., \& Weichselbaum, R. R. (2012). Molecular pathways: Interferon/statl pathway: Role in the tumor resistance to genotoxic stress and aggressive growth. Clinical Cancer Research, 18, 3015-3321.

Malilas, W., Koh, S. S., Kim, S., Srisuttee, R., Cho, I.-R., Moon, J., Yoo, H.-S., Oh, S., Johnston, R. N., \& Chung, Y.-H. (2013). Cancer upregulated gene 2, a novel oncogene, enhances migration and drug resistance of colon cancer cells via STAT1 activation. International Journal of Oncology, 43, 1111-1116.

Mertens, C., Zhong, M., Krishnaraj, R., Zou, W., Chen, X., \& Darnell, J. E., Jr. (2006). Dephosphorylation of phosphotyrosine on STAT1 dimers requires extensive spatial reorientation of the monomers facilitated by the N-terminal domain. Genes \& Development, 20, 3372-3381.

Prieto-Remon, I., Sanchez-Carrera, D., Lopez-Duarte, M., Richard, C., \& Pipaón, C. (2013). Elevated levels of STAT1 in Fanconi anemia group A lymphoblasts correlate with the cells' sensitivity to DNA interstrand crosslinking drugs. Haematologica, 98, 705-713.

Wieczorek, M., Ginter, T., Brand, P., Heinzel, T., \& Krämer, O. H. (2012). Acetylation modulates the STAT signaling code. Cytokine \& Growth Factor Reviews, 23, 293-305.

Cite this article: Göder A, Ginter T, Kröhnert U, Kosan C, Krämer OH (2020). Impact of the STAT1 N-terminal domain for fibrosarcoma cell responses to y-irradiation Experimental Results, 1, e20, 1-9. https://doi.org/10.1017/exp.2020.25 


\section{Peer Reviews}

\section{Reviewing editor: Prof. Martin Michaelis}

University of Kent, School of Biosciences, Canterbury, United Kingdom of Great Britain and Northern Ireland, CT2 7NJ

This article has been accepted because it is deemed to be scientifically sound, has the correct controls, has appropriate methodology and is statistically valid, and met required revisions.

doi:10.1017/exp.2020.25.pr1

\section{Review 1: Impact of the STAT1 N-terminal domain for fibrosarcoma cell responses to Y-irradiation}

Reviewer: Dr. Helmut Pospiech ${ }^{1,2}$ (ID

${ }^{1}$ Leibniz Institute on Aging, PG Biochemistry, Beutenbergstr. 11, Jena, Germany, $07745{ }^{2}$ Oulun Yliopisto, Faculty of Biochemistry and Molecular Medicine, P.O.Box 5400, Oulu, Finland, 90014

Date of review: 12 February 2020

Published online:

(C) The Author(s), 2020. Published by Cambridge University Press This is an Open Access article, distributed under the terms of the Creative Commons Attribution licence (http://creativecommons.org/licenses/by/4.0/), which permits unrestricted re-use, distribution, and reproduction in any medium, provided the original work is properly cited.

Conflict of interest statement. The reviewer shares a secondary affiliation with one of the co-authors of the manuscript.

Comments to the Author: The manuscript represents a congruent set of experimental results. There are some minor issues that should be addressed prior to acceptance:

- Introduce STAT1AAQQ in the methods section

- Fig. 1D. Indicate the number of biological/technical repeats and the statistical test employed

- indicate the number of biological repeats performed for the WB analyses shown (e.g. "The results are representative of $n$ biological repeats")

\section{Score Card}

Presentation

4.3

Is the article written in clear and proper English? (30\%)

Is the data presented in the most useful manner? (40\%)

Does the paper cite relevant and related articles appropriately? (30\%)

Context

Does the abstract correctly embody the content of the article? (25\%)

Does the introduction give appropriate context? (25\%)

Is the objective of the experiment clearly defined? (25\%) 
Analysis

Is the conclusion consistent with the results and discussion? (40\%)

Are the limitations of the experiment as well as the contributions of the experiment clearly outlined? (20\%) 


\section{Review 2: Impact of the STAT1 N-terminal domain for fibrosarcoma cell responses to -irradiation}

Reviewer: Dr. Mark Wass

University of Kent, Biosciences, Canterbury, United Kingdom of Great Britain and Northern Ireland, CT2 7NZ

Date of review: 26 May 2020

Published online:

(c) The Author(s), 2020. Published by Cambridge University Press This is an Open Access article, distributed under the terms of the Creative Commons Attribution licence (http://creativecommons.org/licenses/by/4.0/), which permits unrestricted re-use, distribution, and reproduction in any medium, provided the original work is properly cited.

Conflict of interest statement. Reviewer declares none

Comments to the Author: The manuscript reports thorough piece of work investigating the role of STAT1 in fibrosarcoma. The manuscript is clear and provides findings that will be of interest to the field.

\section{Score Card}

Presentation

5.0

Is the article written in clear and proper English? (30\%)

Is the data presented in the most useful manner? (40\%)

Does the paper cite relevant and related articles appropriately? (30\%)

Context

5.0

Does the title suitably represent the article? (25\%)

Does the abstract correctly embody the content of the article? (25\%)

Does the introduction give appropriate context? (25\%)

Is the objective of the experiment clearly defined? (25\%)

Analysis

Does the discussion adequately interpret the results presented? (40\%)

Is the conclusion consistent with the results and discussion? (40\%)

Are the limitations of the experiment as well as the contributions of the experiment clearly outlined? (20\%) 